Plasmon single- and multi-quantum excitation in free metal clusters as seen by photoelectron spectroscopy

T. Andersson', C. Zhang, A. Rosso, I. Bradeanu, S. Legendre, S. E. Canton, M. Tchaplyguine, G. Öhrwall, S. L. Sorensen, S. Svensson, N. Mårtensson, and O. Björneholm

Citation: The Journal of Chemical Physics 134, 094511 (2011); doi: 10.1063/1.3563751

View online: http://dx.doi.org/10.1063/1.3563751

View Table of Contents: http://aip.scitation.org/toc/jcp/134/9

Published by the American Institute of Physics

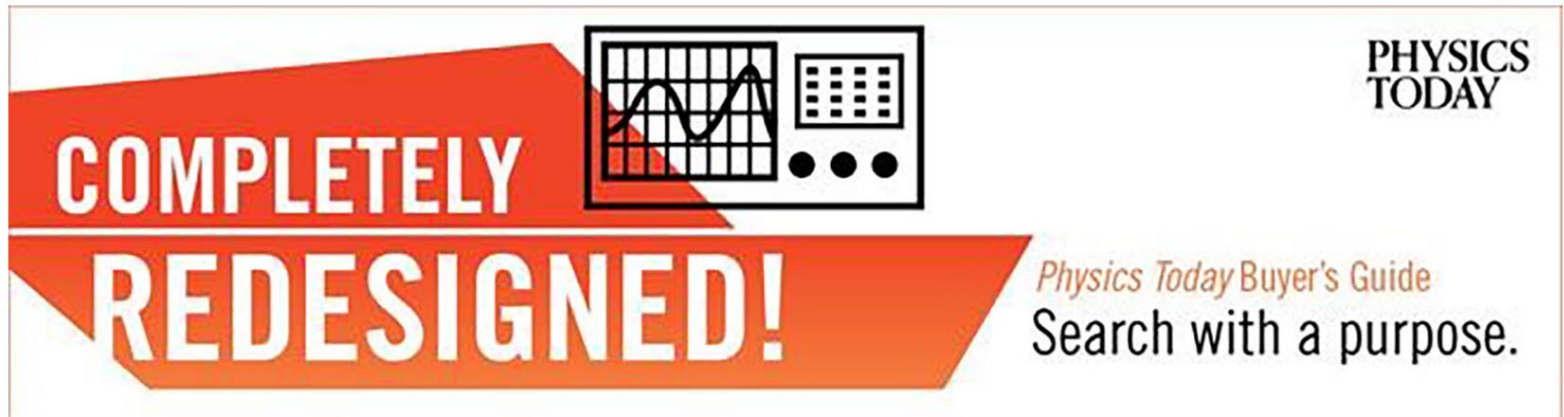




\title{
Plasmon single- and multi-quantum excitation in free metal clusters as seen by photoelectron spectroscopy
}

\author{
T. Andersson, ${ }^{1,2, a)}$ C. Zhang, ${ }^{1}$ A. Rosso, ${ }^{1}$ I. Bradeanu, ${ }^{1}$ S. Legendre, ${ }^{1,3}$ S. E. Canton,,${ }^{3,4}$ \\ M. Tchaplyguine, ${ }^{3}$ G. Öhrwall, ${ }^{3}$ S. L. Sorensen, ${ }^{2}$ S. Svensson, ${ }^{1}$ N. Mårtensson, ${ }^{1,3}$ \\ and O. Björneholm ${ }^{1}$ \\ ${ }^{1}$ Department of Physics and Astronomy, Uppsala University, Box 530, Uppsala, Sweden \\ ${ }^{2}$ Department of Synchrotron Radiation Research, Lund University, Box 118, Lund, Sweden \\ ${ }^{3}$ MAX-lab, Lund University, Box 118, Lund, Sweden \\ ${ }^{4}$ Department of Chemical Physics, Lund University, Box 118, Lund, Sweden
}

(Received 12 July 2010; accepted 21 February 2011; published online 7 March 2011)

\begin{abstract}
Plasmons are investigated in free nanoscale $\mathrm{Na}, \mathrm{Mg}$, and $\mathrm{K}$ metal clusters using synchrotron radiation-based x-ray photoelectron spectroscopy. The core levels for which the response from bulk and surface atoms can be resolved are probed over an extended binding energy range to include the plasmon loss features. In all species the features due to fundamental plasmons are identified, and in $\mathrm{Na}$ and $\mathrm{K}$ also those due to either the first order plasmon overtones or sequential plasmon excitation are observed. These features are discussed in view of earlier results for planar macroscopic samples and free clusters of the same materials. (C) 2011 American Institute of Physics. [doi:10.1063/1.3563751]
\end{abstract}

\section{INTRODUCTION}

Collective electronic phenomena in a solid system with a finite number of constituent atoms is of great interest, both from a fundamental and an applied research perspective. ${ }^{1-3}$ Among such phenomena are plasmons-quantized excitations of the electron density - which are predicted already in the Drude free electron gas model, ${ }^{4}$ the simplest description of metals, known to be a good quantitative approximation for the alkali metals. In studies of free nanoparticles the influence of a substrate on the measurement is avoided. However, the dilute concentrations of such samples present a challenge in terms of experimental sensitivity. In the experimental studies of plasmons in free nanoparticles (or clusters), the most common approach has been the measurement of the optical absorption cross-section in the vicinity of the Mie resonance ${ }^{5}$ known for metallic spheres (see, e.g., Ref. 6). While most studies of this kind were performed on smaller clusters ${ }^{7-9}$ (with a number of constituent atoms below 100), Bréchignac and co-workers ${ }^{10}$ have studied the optical excitation of surface plasmons in free, initially positively charged, potassium clusters in a wide range of sizes up to $\sim 900$ constituent atoms. They found the surface plasmon energy to be below the Mie resonance (which is $\sqrt{3}$ times smaller than the bulk plasmon frequency) and it was shown to increase slowly with the cluster size. Implementing the optical absorption method for studying bulk plasmons is problematic, both from a theoretical and an experimental point of view. ${ }^{4,11}$ Using $\mathrm{x}$-ray photoelectron spectroscopy (XPS), the observation of bulk plasmons on metallic macroscopic samples is, however, known to be achievable. ${ }^{12}$ The sudden appearance of the electron-hole pair (due to the core-level ionization) and

\footnotetext{
a) Author to whom correspondence should be addressed. Electronic mail: Tomas.Andersson@maxlab.lu.se.
}

photoelectron scattering can excite plasmons in the interior as well as at the surface. In the planar-sample case the surface plasmon energy is predicted by the theory to be $\sqrt{2}$ times smaller than the bulk plasmon energy, both for the optical and electron scattering excitation. ${ }^{4,13}$ For electron scattering, in macroscopic alkali and alkali-earth metal $(\mathrm{Na}, \mathrm{K}, \mathrm{Mg})$ samples, this has been confirmed through XPS and electron energy loss spectroscopy experiments. ${ }^{12,14,15}$

In XPS, due to the losses of kinetic energy on the plasmon excitation, the response of the photoelectrons appears in the spectra as broadened replicas of the main features, shifted by the plasmon excitation energy toward lower kinetic energies. Recently, we presented a photoelectron spectroscopy study on free, initially neutral, potassium clusters consisting of a few thousand atoms. ${ }^{16}$ For these clusters both the surface and bulk plasmon excitation energies were found to be indistinguishably close to those of the "infinite" planar solid case. In the present work, we expand our investigation to include clusters of sodium and magnesium. As earlier for $\mathrm{K}$ clusters, ${ }^{16}$ in the nanometer size range of this experiment, for $\mathrm{Na}$ and $\mathrm{Mg}$, the plasmon excitation energies for both bulk and surface have been found to be approximately equal (to within $\sim 5 \%$ ) to the experimental values of the corresponding macroscopic solids. Due to the relatively low plasmon excitation energies of sodium and potassium clusters we have been able to observe also the energy loss features corresponding to excitations of two plasmon quanta.

\section{EXPERIMENTAL}

\section{A. Experimental setup and procedure}

The clusters were produced using a gas aggregation source as described in Ref. 17. A resistively heated oven was placed inside a liquid nitrogen-cooled cryostat. Solid 
samples of the metals to be studied were vaporized in the oven. Argon was let into the cryostat at a pressure of about 2 mbars to (1) cool the vapor atoms through collisions and let them condense into clusters, and (2) transfer the clusters out of the cryostat into the experimental ionization chamber. A nozzle of a Laval-like profile with a $1.5 \mathrm{~mm}$ throat diameter at the cryostat exit shaped a cluster beam that propagated perpendicular to the synchrotron light. The oven was heated to temperatures creating partial pressures of $10^{-1}-10^{-2}$ mbar. Similar operation conditions for all three metals (partial vapor pressure, cryostat temperature, and Ar input pressure) created cluster size distributions centered at different mean size-values, $\langle N\rangle$, due to varying clusterization efficiency for the different elements. The experiments were performed at the synchrotron radiation facility MAX-lab, at the soft x-ray beamline I411. ${ }^{18}$ The photoelectron spectra were recorded using a Scienta R4000 electron energy analyzer, mounted perpendicular both to the horizontally polarized synchrotron light and to the cluster beam. For $\mathrm{Na}, \mathrm{Mg}$, and $\mathrm{K}$ clusters, the shallowest core levels, for which the response from bulk and surface atoms can be resolved-2p for $\mathrm{Na}$ and $\mathrm{Mg}, 3 \mathrm{p}$ for $\mathrm{K}$-were probed. The absolute energy calibration was performed using argon lines present in the spectra due to the uncondensed inert-gas atoms in the cluster beam. The binding energy scales in all spectra refer to the vacuum level. The $\mathrm{Na}, \mathrm{Mg}$, and $\mathrm{K}$ clusters were irradiated by light with 80, 80, and $119.5 \mathrm{eV}$ photon energies, respectively. These energies were chosen to get optimal recording conditions.

\section{B. Spectral analysis: Main features, fundamental plasmons, plasmon overtone region}

Typical core level photoelectron spectra for free $\mathrm{K}, \mathrm{Na}$, and $\mathrm{Mg}$ clusters are presented in Figs. 1-3. The measured energy range includes the energy loss features due to the fundamental plasmon excitations ${ }^{16}$ and in the case of sodium and potassium extends above the energy interval where the loss features corresponding to the first order plasmon overtones (see below for details) can be expected.

The close similarity between the cluster and solid spectra implies that the clusters are large enough to be considered metallic. In this case a cluster can be treated as a uniform conducting sphere (metal sphere approximation) and its mean radius, $R$, can be estimated from the difference in binding energy compared to the solid-state case. ${ }^{17,19-22}$ The K clusters are estimated to have a mean radius of $\sim 5 \mathrm{~nm}$ and to contain between $10^{3}$ and $10^{4}$ atoms. For $\mathrm{Na}$ the mean cluster radius and average number of constituent atoms are estimated to about $3 \mathrm{~nm}$ and $\sim 10^{3}$, respectively. The $\mathrm{Mg}$ clusters are estimated to be quite large, with radii above $10 \mathrm{~nm}$ thus containing some hundred thousand atoms. The main uncertainty comes from the discrepancy in the literature data on the work functions of the corresponding solid materials.

Since the shapes of the main cluster features resemble those of the corresponding solid, the features are assumed to be a sum of the same contributions as in the solid spectrum, namely, of the bulk and surface contributions from each of the two spin-orbit states. As in our previous publication, ${ }^{16}$ the main features are fitted with four Doniach-Šunjić profile
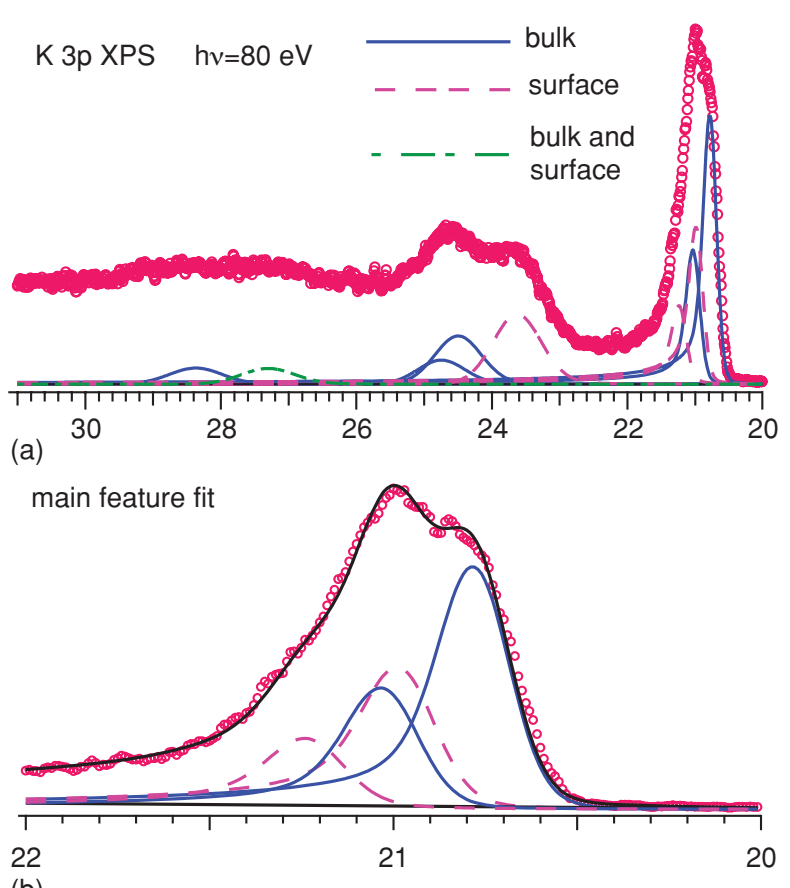

(b)

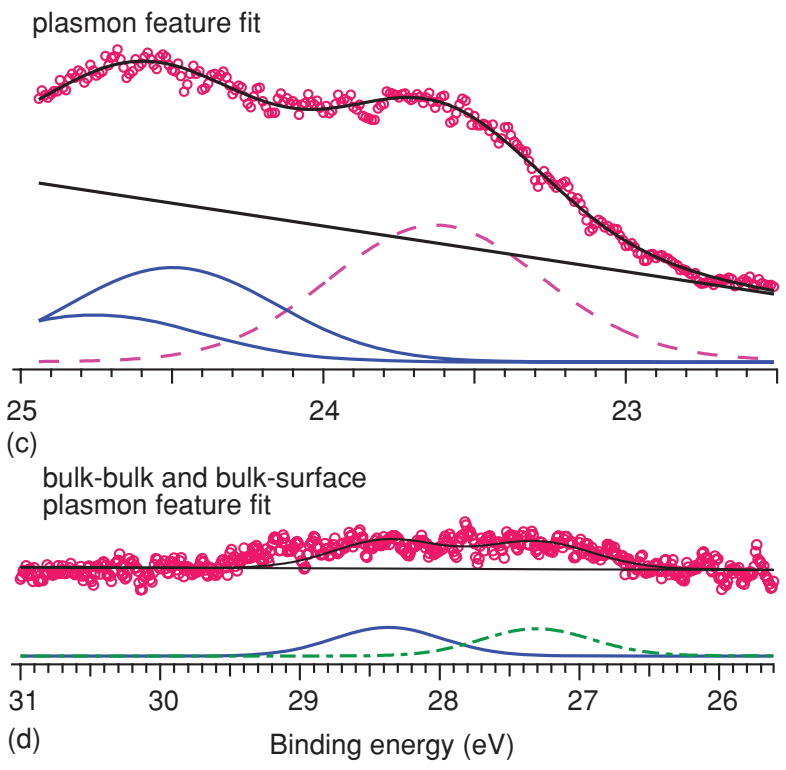

FIG. 1. XPS of the $3 p$ level in K clusters. (a) Overview of the whole investigated binding energy range. (b) The fit of the $3 p$ main feature. (c) The fit of the plasmon features. (d) The fit of the bulk-bulk and bulk-surface plasmon features. The full blue lines in the spectral decomposition represent the bulk photoelectrons, bulk and "bulk-bulk" plasmons, whereas the dashed pink lines are due to the surface electrons and surface plasmons. The green point-dashed line is the bulk-surface plasmons. The full black straight lines are backgrounds, and the sums of all curves (close-to-data lines) are also black. The curves resulting from the fits (b)-(d) have been copied into the overview spectrum (a). Note that both $x$ - and $y$-scales differ between the spectra. Binding energies are relative to the vacuum level. A constant background has been subtracted in the (d)-spectrum.

peaks. Some of the parameter values of these peaks have been fixed to the solid-state values from the literature. The main feature parameters used in (or obtained from) the fits are given in Table I.

Assuming that surface plasmons can be excited by all photoelectrons, and that observable bulk plasmon losses are experienced mostly by electrons from the bulk, the bulk and 


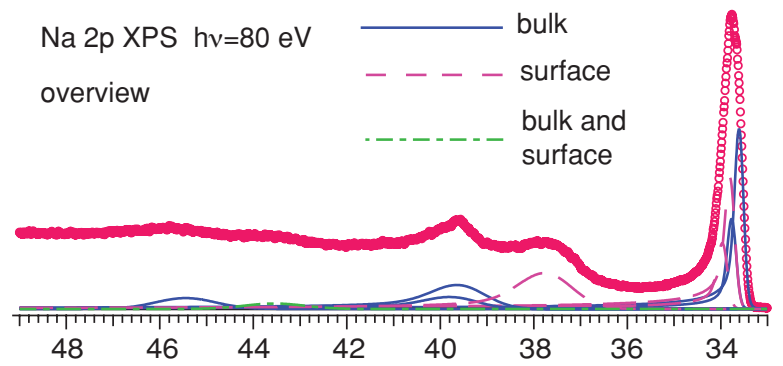

(a)

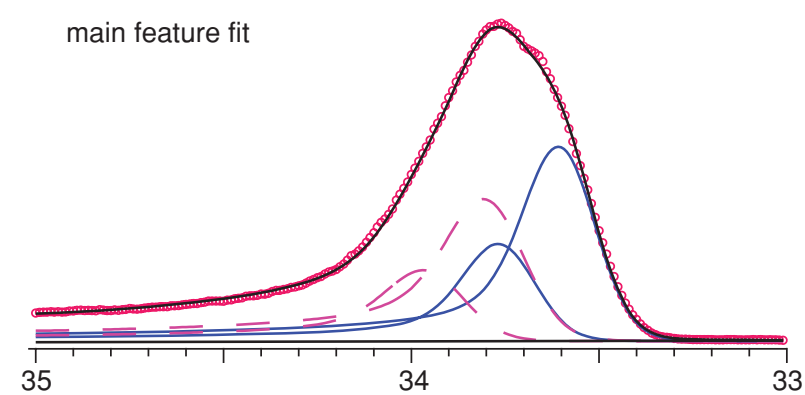

(b)
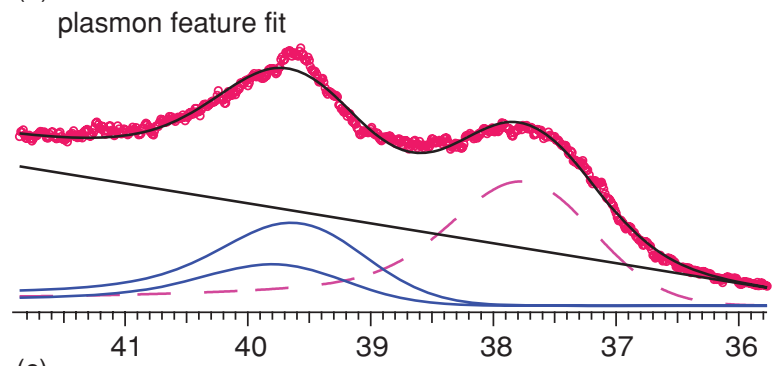

(c)

bulk-bulk and bulk-surface plasmon feature fit

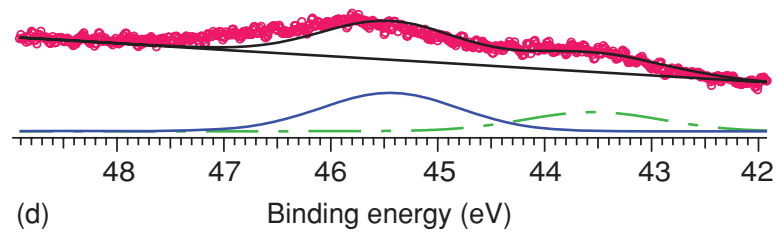

FIG. 2. XPS of the $2 p$ level in Na clusters. (a) Overview of the whole investigated binding energy range. (b) The fit of the $2 p$ main feature. (c) The fit of the plasmon features. (d) The fit of the bulk-bulk and bulk-surface plasmon features. The full blue lines in the spectral decomposition represent the bulk photoelectrons, bulk and bulk-bulk plasmons, whereas the dashed pink lines are due to the surface electrons and surface plasmons. The green pointdashed line is the bulk-surface plasmons. The full black straight lines are backgrounds, and the sums of all curves (close-to-data lines) are also black. The curves resulting from the fits (b)-(d) have been copied into the overview spectrum (a). Note that both $x$ - and $y$-scales differ between the spectra. Binding energies are relative to the vacuum level. Constant backgrounds have been subtracted in the (c)- and (d)-spectra.

surface fundamental plasmon features were fitted with two and four different peaks, respectively. Because of the small spin-orbit and bulk-surface splittings $(0.1-0.3 \mathrm{eV})$ compared to the widths of the plasmon peaks and the energy difference between them, we have chosen to fit the fundamental plasmon features with three peaks: two spin-orbit split peaks for the bulk and one common for the four surface plasmon contributions.

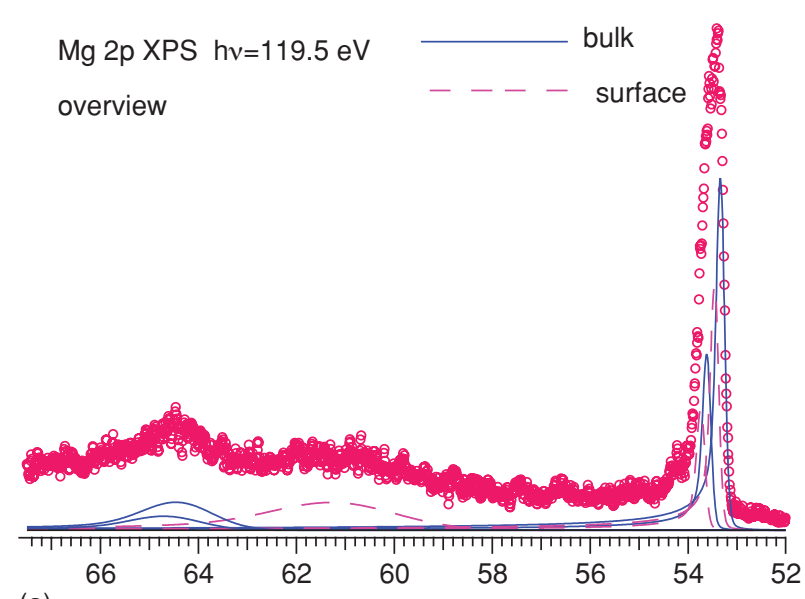

(a)

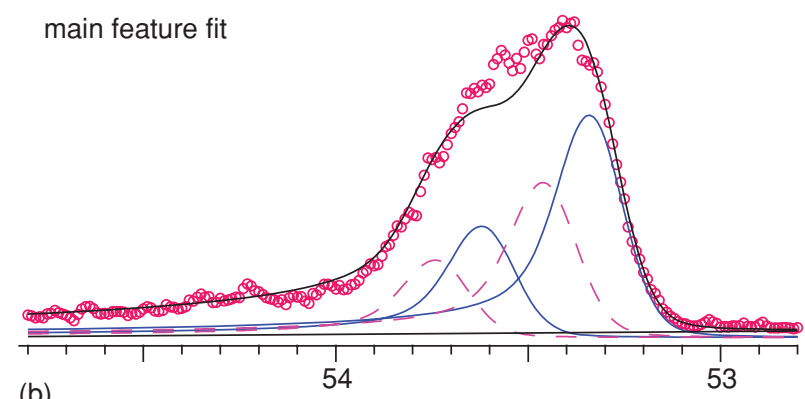

(b)

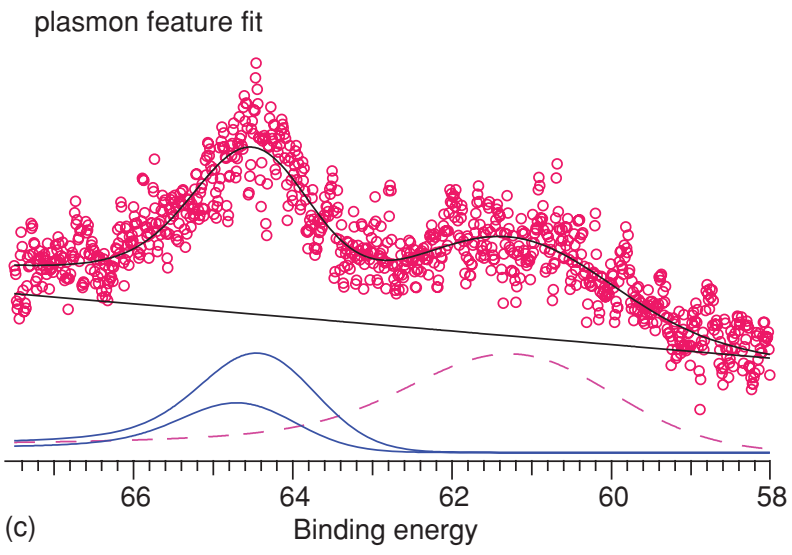

FIG. 3. XPS of the $2 p$ level in Mg clusters. (a) Overview of the whole investigated binding energy range. (b) The fit of the $2 p$ main feature. (c) The fit of the plasmon features. The full blue lines in the spectral decomposition represent the bulk photoelectrons and bulk plasmons, whereas the dashed pink lines are due to the surface electrons and surface plasmons. The full black straight lines are backgrounds, and the sums of all curves (close-to-data lines) are also black. The curves resulting from the fits (b) and (c) have been copied into the overview spectrum (a). Note that both $x$ and $y$ scales differ between the spectra. Binding energies are relative to the vacuum level. A constant background has been subtracted in the (c)- spectrum.

In the core-level photoelectron spectra of many macroscopic metals, energy loss features interpreted to arise from the harmonic excitation of plasmon overtones, have been observed. ${ }^{14,28}$ In the present case, for $\mathrm{K}$ and Na clusters, we observe features in the corresponding energy region. These features appear on top of a complex background, which makes fitting of the spectrum challenging. Based on the solid-state results, and under the assumption of harmonic overtone excitation, two main peaks are expected: one 
TABLE I. Main feature parameter values.

\begin{tabular}{|c|c|c|c|c|c|c|c|c|c|c|c|c|}
\hline & \multicolumn{4}{|c|}{$\mathrm{Na} 2 \mathrm{p}$ Cluster $\langle N\rangle \sim 10^{3}$} & \multicolumn{4}{|c|}{$\operatorname{Mg} 2$ p Cluster $\langle N\rangle \sim 10^{5}$} & \multicolumn{4}{|c|}{$\mathrm{K} 3 \mathrm{p}$ Cluster $\langle N\rangle \sim 10^{3}-10^{4}$} \\
\hline & \multicolumn{2}{|c|}{ Bulk } & \multicolumn{2}{|c|}{ Surface } & \multicolumn{2}{|c|}{ Bulk } & \multicolumn{2}{|c|}{ Surface } & \multicolumn{2}{|c|}{ Bulk } & \multicolumn{2}{|c|}{ Surface } \\
\hline & $3 / 2$ & $1 / 2$ & $3 / 2$ & $1 / 2$ & $3 / 2$ & $1 / 2$ & $3 / 2$ & $1 / 2$ & $3 / 2$ & $1 / 2$ & $3 / 2$ & $1 / 2$ \\
\hline Binding energy $(\mathrm{eV})$ & $33.6(1)^{\mathrm{a}}$ & $33.8(1)^{\mathrm{a}}$ & $33.8(1)^{\mathrm{a}}$ & $33.9(1)^{\mathrm{a}}$ & $53.3(1)^{\mathrm{a}}$ & $53.6(1)^{\mathrm{a}}$ & $53.4(1)^{\mathrm{a}}$ & $53.7(1)^{\mathrm{a}}$ & $20.8(1)^{\mathrm{a}}$ & $21.0(1)^{\mathrm{a}}$ & $21.0(1)^{\mathrm{a}}$ & $21.2(1)^{\mathrm{a}}$ \\
\hline Lorentzian width $(\mathrm{eV})$ & \multicolumn{4}{|c|}{$0.01^{\mathrm{b}}$} & \multicolumn{4}{|c|}{$0.03(1)^{\mathrm{a}}$} & $0.02^{\mathrm{c}}$ & $0.03^{\mathrm{c}}$ & $0.04^{\mathrm{c}}$ & $0.06^{\mathrm{c}}$ \\
\hline Gaussian width (eV) & \multicolumn{2}{|c|}{$0.21(1)^{\mathrm{a}}$} & \multicolumn{2}{|c|}{$0.22(1)^{\mathrm{a}}$} & \multicolumn{4}{|c|}{$0.18(1)^{\mathrm{a}}$} & \multicolumn{2}{|c|}{$0.21(1)^{\mathrm{a}}$} & \multicolumn{2}{|c|}{$0.22(1)^{\mathrm{a}}$} \\
\hline Singularity index & \multicolumn{2}{|c|}{$0.16^{\mathrm{d}}$} & \multicolumn{2}{|c|}{$0.22^{\mathrm{d}}$} & \multicolumn{4}{|c|}{$0.16^{\mathrm{e}}$} & 0.1 & & \multicolumn{2}{|c|}{$0.19^{c}$} \\
\hline Bulk-surface shift (eV) & \multicolumn{4}{|c|}{$0.19^{\mathrm{d}}$} & \multicolumn{4}{|c|}{$0.12(1)^{\mathrm{f}}$} & \multicolumn{4}{|c|}{$0.20^{\mathrm{c}}$} \\
\hline Spin-orbit shift (eV) & \multicolumn{4}{|c|}{$0.16^{\mathrm{d}}$} & \multicolumn{4}{|c|}{$0.28^{\mathrm{d}}$} & \multicolumn{4}{|c|}{$0.25^{\mathrm{c}}$} \\
\hline
\end{tabular}

${ }^{\mathrm{a}}$ Values taken from the fit.

${ }^{\mathrm{b}}$ Reference 23.

${ }^{\mathrm{c}}$ Reference 24

${ }^{\mathrm{d}}$ Reference 25

${ }^{\mathrm{e}}$ Varied around the value from Ref. 26

${ }^{\mathrm{f}}$ Varied around the value from Ref. 27.

separated from the main feature by twice the surface plasmon energy ("surface-surface" plasmon), the other by twice the bulk plasmon energy ("bulk-bulk" plasmon). Indeed, a clear feature is observed at about the bulk-bulk plasmon position for both $\mathrm{Na}$ and $\mathrm{K}$ clusters. However, in both cases ( $\mathrm{K}$ and $\mathrm{Na}$ ), no peaks are discernible at the position expected for the surface-surface plasmons. Instead, a peak energetically separated from the main features by a sum of one bulk plasmon and one surface plasmon quantum (denoted further as "bulk-surface" plasmon) is observed. Such features have not been reported earlier for the planar-sample case and cannot be explained by harmonic excitation. In the fitting procedure all these peaks have been given fixed widths that appeared reasonable. These widths as well as those for the fundamental plasmon peaks are given in Table II. The origin of the solidlike response and the nonconventional energy loss features in the overtone region of the core-level photoelectron spectra are discussed further in Sec. III.

\section{DISCUSSION}

In the present study, we address (1) the cluster-size dependence of the fundamental plasmon energies for two new (in addition to $\mathrm{K}^{16}$ ) materials in a size range not far from typical nanoparticles, from a few to some tens of nanometers, as well as the differences to the optically excited plasmons in spherical clusters; and (2) the appearance of loss features in free clusters in the energy region where harmonic plasmon overtones have been observed in the planar macroscopic case. Concerning the first issue we have been motivated by reports

TABLE II. Plasmon loss-feature total peak widths.

\begin{tabular}{lcccc}
\hline \hline & & $\mathrm{Na}$ & $\mathrm{Mg}$ & $\mathrm{K}$ \\
\hline Fundamental plasmon & Surface & $1.3(4)$ & $2.8^{\mathrm{a}}$ & $0.8(2)$ \\
feature widths (eV) & Bulk & $1.3(4)$ & $1.6(4)$ & $0.8(2)$ \\
& & & & $0.9(2)$ \\
Plasmon overtone or sequential & Surface-bulk & $1.5(4)$ & & $0.9(2)$ \\
excitation feature widths (eV) & Bulk-bulk & $1.5(4)$ & & 0.96 \\
\hline \hline
\end{tabular}

${ }^{a}$ With the present signal-to-noise ratio the accuracy of the estimate is considerably lower than in the other cases. on the change of the surface and bulk optical plasmon energies in clusters with dimensions in the range of $10^{2}-10^{3}$ atoms (see, e.g., Refs. 10 and 11). In view of the experimental novelty, however, the multiquantum plasmon excitation will be our first point of discussion.

As mentioned above, in photoelectron spectroscopy experiments $^{12,28}$ on supported macroscale planar solids, the appearance of harmonic regularly spaced energy loss features has been attributed to harmonic plasmon overtone excitation. For free clusters with a different, close-to-spherical geometry and a countable number of valence electrons the possibility to excite a multiple quanta of one and the same plasmon oscillation is not obvious, and if an overtone excitation is possible, it is not necessarily harmonic. ${ }^{6,29}$ Experimental studies of plasmon multiquantum-excitations in free clusters were hindered by the absence of adequate high photonenergy light sources capable of probing dilute samples. The recent availability of high-power femtosecond lasers allowing multiphoton excitation, has given some insight into the plasmon overtone-problem in free clusters. ${ }^{30}$ In Ref. 30 positively charged, mass-selected sodium clusters consisting of just less than 100 atoms have been studied, and a change in the cluster fragmentation pattern has been interpreted as due to harmonic plasmon excitation in the clusters. However, only the surface electron density oscillations, which define the optical absorption, could be probed.

In the photoelectron spectra of the present work, features which can be interpreted as due to harmonic plasmon excitation have been observed. Indeed, a satisfactory fit of the spectrum is obtained (Figs. 1 and 2) with the assumption that the first "bulk-bulk" loss features are separated from the main features by twice the energy of the fundamental bulk plasmons. However, the experimental observation of well-defined features in the bulk-surface plasmon spectral region indicates the importance of sequential processes. We interpret it as due to the loss of kinetic energy, first due to a bulk plasmon excitation followed by a surface plasmon excitation. This implies that a part of the intensity in the bulk-bulk plasmon could be attributed to sequential electron scattering events in the bulk. The attenuation of the flux of photoelectrons on their 
TABLE III. Plasmon excitation energies. In this work, bulk plasmon values have been taken as the difference between one of the bulk plasmon peaks and its corresponding main feature bulk peak. Surface plasmon values have been calculated as the surface plasmon peak energy and the energy halfway between the main feature peaks that are highest and lowest in binding energy.

\begin{tabular}{|c|c|c|c|c|c|c|}
\hline & \multicolumn{2}{|c|}{$\mathrm{Na}\left(\right.$ Cluster $\left.\langle N\rangle \sim 10^{3}\right)$} & \multicolumn{2}{|c|}{$\operatorname{Mg}\left(\right.$ Cluster $\left.\langle N\rangle \sim 10^{5}\right)$} & \multicolumn{2}{|c|}{$\mathrm{K}\left(\right.$ Cluster $\left.\langle N\rangle \sim 10^{3}-10^{4}\right)$} \\
\hline & Bulk & Surface & Bulk & Surface & Bulk & Surface \\
\hline Drude model solid values $(\mathrm{eV})$ & 6.0 & 4.3 & 10.9 & 7.7 & 4.4 & 3.1 \\
\hline Solid exp. values (eV) & $5.9^{\mathrm{a}}$ & $4.0^{\mathrm{b}}$ & $10.8^{\mathrm{a}}$ & $7.3^{\mathrm{a}}$ & $3.9^{\mathrm{c}}$ & $2.7^{\mathrm{b}}$ \\
\hline Cluster exp. values (this work) (eV) & $5.9(2)$ & $3.9(2)$ & $11.0(4)$ & $7.5(4)$ & $3.7(2)$ & $2.6(2)$ \\
\hline
\end{tabular}

aReference 14

${ }^{\mathrm{b}}$ Reference 15 .

${ }^{\mathrm{c}}$ Reference 28

way out through the sample bulk and surface is known to be very efficient (evidenced by the probing depth of XPS on the order of a few monolayers) especially for the kinetic energies in question. In the present size range the clusters consist of just a few spherical "layers," and the fraction of surface atoms is large. This is a likely explanation of the substantial surface and bulk-surface plasmon intensity. The plasmon-tomain feature intensity ratio indeed bears witness that also for clusters the plasmon excitation is one of the efficient attenuation mechanisms for the photoelectrons.

Returning to the rationalization of the observed fundamental plasmon energies, recollect that for the metals under investigation, the Drude theory predicts solid-state plasmon energies quantitatively close to those measured experimentally for solids and for clusters in the present size range $\left(\langle N\rangle \sim 10^{3}-10^{5}\right)$. According to the Drude model, the bulk plasmon energy, $E_{\mathrm{bp}}$, is proportional to the square root of the electron density, $n$, and is given by

$$
E_{\mathrm{bp}}=e h \sqrt{\frac{n}{\pi m_{e}}},
$$

where $m_{e}$ is the electron mass. ${ }^{4}$ For macroscopic $\mathrm{Na}$ and $\mathrm{Mg}$, the plasmon energies given by formula (1) deviate by less than $10 \%$ from the solid experimental values (see Table III). For macroscopic K, the differences from the observed values are larger but still less than $15 \%$. With respect to the considerations above, the practically identical solid and cluster plasmon energies mean that the electron density in clusters of the present size range is close to that of the corresponding macroscale substance despite possible differences in geometric structure. For the surface plasmons in close-to-spherical clusters, the question arises as to why the collective electron reaction to optical radiation and to core-hole ionization differs. More specifically, why is it that at the spherical surfaces of clusters, the external electromagnetic field (optical radiation) excites collective electron oscillations with a frequency around the Mie resonance or below, while the internal fielddue to the electron-hole pair appearance-causes electron oscillations with a frequency close to that of the planar solid.

\section{SUMMARY}

Using a gas aggregation source, we have produced free, neutral clusters of sodium, magnesium, and potassium in the size range of $\sim 10^{3}-10^{5}$ atoms. The shallowest core levels for which the response from bulk and surface atoms can be resolved by means of XPS have been probed over an extended binding energy interval that included the plasmon loss features. In all cases the spectral features closely resemble those of the solid. The fundamental bulk and surface plasmon excitation energies are found to be approximately the same as the experimental values for the supported planar solid samples. To the best of our knowledge, bulk plasmons have not earlier been unambiguously detected in free $\mathrm{Na}$ and $\mathrm{Mg}$ clusters of sizes up to the present range. In addition, for $\mathrm{Na}$ and $\mathrm{K}$ clusters, features due to photoelectrons that have lost energies corresponding to the sum of two fundamental plasmon quanta have been observed. Similar features in the photoelectron spectra of macroscopic samples have been interpreted as harmonic plasmon overtones. In the present work, also sequential excitation of one bulk and one surface plasmon has been suggested as an explanation for the spectral features in $\mathrm{Na}$ and $\mathrm{K}$ clusters detected at the corresponding energies. This also means that a significant part of the intensity in the "bulkbulk" region can be due to two sequential events. One cannot resolve sequential and harmonic excitations in our experiment, so additional investigations of the relevant issues are desirable. Another question on which more work could shed some light is the absence of the typical Mie plasmons in the collective reaction of the spherical-surface electrons to the electron-impact excitation following the core-level ionization.

\section{ACKNOWLEDGMENTS}

The authors would like to acknowledge the financial support from the Swedish Research Council (VR), the Göran Gustafsson Foundation, the Knut and Alice Wallenberg Foundation, the Crafoord Foundation, Nordforsk, and the Swedish Foundation for Strategic Research. The authors would also like to thank the MAX-lab staff for their assistance during the experiments.

${ }^{1}$ J. A. Schwarz, C. Contescu, and K. Putyera, Dekker Encyclopedia of Nanoscience and Nanotechnology, 2nd ed. (Marcel Dekker Inc., New York, 2008).

${ }^{2}$ A. M. Schwartzberg and J. Z. Zhang, J. Phys. Chem. 112, 10323 (2008).

${ }^{3}$ F. J. García de Abajo, Rev. Mod. Phys. 82, 209 (2010).

${ }^{4}$ N. W. Ashcroft and N. D. Mermin, Solid State Physics (Harcourt, Orlando, FL, 1976).

${ }^{5}$ G. Mie, Ann. Phys. 330, 377 (1908).

${ }^{6}$ K. Kolwas, S. Demianiuk, and M. Kolwas, J. Phys. B 29, 4761 (1996). 
${ }^{7}$ W. A. de Heer, K. Selby, V. Kresin, J. Masui, M. Vollmer, A. Châteland, and W. D. Knight, Phys. Rev. Lett. 97, 1805 (1987).

${ }^{8}$ K. Selby, V. Kresin, J. Masui, M. Vollmer, W. A. de Heer, A. Scheidemann, and W. D. Knight, Phys. Rev. B 43, 4565 (1991).

${ }^{9}$ Th. Reiners, Ch. Ellert, M. Schmidt, and H. Haberland, Phys. Rev. Lett. 74, 1558 (1995).

${ }^{10}$ C. Bréchignac, P. Cahuzac, N. Kebaïli, J. Leygnier, and A. Sarfati, Phys. Rev. Lett. 68, 3916 (1992).

${ }^{11}$ C. Xia, C. Yin, and V. V. Kresin, Phys. Rev. Lett. 102, 156802 (2009).

${ }^{12} \mathrm{~S}$. Hüfner, Photoelectron Spectroscopy (Springer Verlag, Germany, 1995).

${ }^{13}$ A. C. Simonsen, F. Yubero, and S. Tougaard, Phys. Rev. B 56, 1612 (1997).

${ }^{14}$ P. Steiner, H. Höchst, and S. Hüfner, Z. Phys. B 30, 129 (1978).

${ }^{15}$ K.-D. Tsuei, E. W. Plummer, A. Liebsch, E. Pehlke, K. Kempa, and P. Bakshi, Surf. Sci. 247, 302 (1991).

${ }^{16}$ A. Rosso, G. Öhrwall, I. L. Bradeanu, S. Svensson, O. Björneholm, and M. Tchaplyguine, Phys. Rev. A 77, 043202 (2008).

${ }^{17}$ S. Peredkov, G. Öhrwall, J. Schulz, M. Lundwall, T. Rander, A. Lindblad, H. Bergersen, A. Rosso, W. Pokapanich, N. Mårtensson, S. Svensson, S. L. Sorensen, O. Björneholm, and M. Tchaplyguine, Phys. Rev. B 75, 235407 (2007).

${ }^{18}$ M. Bässler, A. Ausmees, M. Jurvansuu, R. Feifel, J.-O. Forsell, P. de Tarso Fonseca, A. Kivimäki, S. Sundin, S. L. Sorensen, R. Nyholm, O. Björne- holm, S. Aksela, and S. Svensson, Nucl. Instrum. Methods Res. A 469, 382 (2001).

${ }^{19}$ G. Makov, A. Nitzan, and L. E. Brus, J. Chem. Phys. 88, 5076 (1988).

${ }^{20}$ M. A. Hoffmann, G. Wrigge, and B. von Issendorff, Phys. Rev. B 66, 041401 (2002).

${ }^{21}$ V. Senz, T. Fischer, P. Oelßner, J. Tiggesbäumker, J. Stanzel, C. Bostedt, H. Thomas, M. Schöffler, L. Foucar, M. Martins, J. Neville, M. Neeb, Th. Möller, W. Wurth, E. Rühl, R. Dörner, H. Schmidt-Böcking, W. Eberhardt, G. Ganteför, R. Treusch, P. Radcliffe, and K.-H. Meiwes-Broer, Phys. Rev. Lett. 102, 138303 (2009).

${ }^{22}$ M. Seidl, J. P. Perdew, M. Brajczewska, and C. Fiolhais, Phys. Rev. B 55 13288 (1997).

${ }^{23}$ T. A. Callcott, E. T. Arakawa, and D. L. Ederer, Phys. Rev. B 18, 6622 (1978).

${ }^{24}$ G. K. Wertheim and D. M. Riffe, Phys. Rev. B 52, 14906 (1995).

${ }^{25}$ G. K. Wertheim, D. M. Riffe, and P. H. Citrin, Phys. Rev. B 45, 8703 (1992).

${ }^{26}$ P. H. Citrin, G. K. Wertheim, and Y. Baer, Phys. Rev. B 16, 4256 (1977).

${ }^{27}$ R. Kammerer, J. Barth, F. Gerken, C. Kunz, S. A. Flodström, and L. I. Johansson, Phys. Rev. B 26, 3491 (1982).

${ }^{28}$ G. Pirug, A. Winkler, and H. P. Bonzel, Surf. Sci. 163, 153 (1985).

${ }^{29}$ W. de Heer, Rev. Mod. Phys. 65, 611 (1993).

${ }^{30}$ R. Schlipper, R. Kusche, B. von Issendorff, and H. Haberland, Phys. Rev. Lett. 80, 1194 (1998). 